\title{
The transcription factor FLC confers a flowering response to vernalization by repressing meristem competence and systemic signaling in Arabidopsis
}

\author{
Iain Searle, ${ }^{1}$ Yuehui He, ${ }^{2,3}$ Franziska Turck, ${ }^{1}$ Coral Vincent, ${ }^{1}$ Fabio Fornara, ${ }^{1}$ Sandra Kröber, ${ }^{1}$ \\ Richard A. Amasino, ${ }^{2}$ and George Coupland ${ }^{1,4}$ \\ ${ }^{1}$ Max Planck Institute for Plant Breeding, D-50829 Cologne, Germany; ${ }^{2}$ Department of Biochemistry, University \\ of Wisconsin-Madison, Madison, Wisconsin 53706, USA
}

\begin{abstract}
Floral development at the Arabidopsis shoot apical meristem occurs in response to environmental cues that are perceived in different tissues. Photoperiod is detected in the vascular tissue of the leaf (phloem) and promotes production of a systemic signal that induces flowering at the meristem. Vernalization, the response to winter temperatures, overcomes a block on photoperiodic floral induction. In Arabidopsis, this block is caused by inhibitors of flowering that comprise several related MADS-box transcription factors, the most prominent of which is FLC. We show that FLC delays flowering by repressing production in the leaf of at least two systemic signals, one of which is controlled by the RAF kinase inhibitor-like protein FT. Reducing expression of these signals indirectly represses expression of genes involved in floral induction at the meristem. In addition, FLC expression in the meristem impairs response to the FT signal by directly repressing expression of the SOC1 MADS-box transcription factor and preventing up-regulation of the bZIP transcription factor FD. Repression of genes acting at multiple levels in this hierarchy is required for the extreme delay in flowering caused by FLC. An FLC:HA fusion protein binds directly in vivo to the promoter regions of $F D$ and $S O C 1$ and to the first intron of $F T$. Thus vernalization relieves transcriptional repression of key regulatory genes in both the leaf and meristem, allowing production of systemic signals in the leaves and conferring competence on the meristem to respond to these signals.
\end{abstract}

[Keywords: Arabidopsis; flowering; photoperiod; vernalization]

Supplemental material is available at http://www.genesdev.org.

Received November 18, 2005; revised version accepted January 31, 2006.

In Arabidopsis, reproductive development is initiated by the formation of floral primordia on the flanks of the shoot apical meristem. The time at which flowering occurs is governed by environmental cues such as day length and temperature, and is influenced by endogenous signals related to the age of the plant. Classical physiological experiments demonstrated that environmental signals that influence flowering are perceived in different tissues. For example, day length is detected in expanded leaves, and in response to exposure to day lengths that trigger flowering, a systemic signal termed the floral stimulus travels through the phloem to the shoot apical meristem, where it induces floral development (Knott 1934; Zeevaart 1976; Corbesier and Coupland 2005). Ver-

${ }^{3}$ Present address: Department of Biological Sciences, Faculty of Science, National University of Singapore, Science Drive 4, Singapore 117543. ${ }^{4}$ Corresponding author.

E-MAIL coupland@mpiz-koeln.mpg.de; FAX 49-221-5062207.

Article and publication are at http://www.genesdev.org/cgi/doi/10.1101/ gad.373506. nalization, the acquisition of competence to flower that results from exposure to extended periods of low temperatures that mimic winter conditions, typically relieves a block to the photoperiod pathway (Lang 1965). Here we describe an analysis of the tissues in which key Arabidopsis genes involved in vernalization act to control flowering and demonstrate the significance of transcriptional regulation in the phloem and the meristem for the vernalization response.

Genetic analyses of the control of flowering in Arabidopsis thaliana identified four major floral promotion pathways (Mouradov et al. 2002; Boss et al. 2004). Environmental responses studied genetically in Arabidopsis include photoperiodic control of flowering, induction of flowering by response to long days (LD) of spring or early summer, and vernalization, which is the promotion of flowering by extended exposures to low temperatures that mimic winter conditions. The responses to day length (or photoperiod) and vernalization are mediated by pathways that specifically control responses to 
these environmental cues, whereas the autonomous and gibberellin (GA) pathways appear to act independently of these signals (Mouradov et al. 2002; Boss et al. 2004). However, these pathways converge to regulate the transcription of a set of integrator genes, particularly LEAFY (LFY), SUPPRESSOR OF OVEREXPRESSION OF CONSTANS (SOC1), and FLOWERING LOCUS T (FT) (Blazquez and Weigel 2000; Lee et al. 2000; Samach et al. 2000; Michaels et al. 2005).

CONSTANS (CO) and FT were placed in the photoperiod pathway because mutations in these genes delay flowering under LDs, but not under short days (SDs), and thereby modulate the response to photoperiod (Koornneef et al. 1991). Expression of CO, which encodes a zinc finger protein that regulates transcription, is controlled at the transcriptional level by the circadian clock and exposure to light, and at the post-transcriptional level by stabilization of the protein in response to light (Putterill et al. 1995; Suarez-Lopez et al. 2001; Imaizumi et al. 2003; Valverde et al. 2004). The combination of these regulatory mechanisms results in accumulation of $\mathrm{CO}$ protein specifically under LDs, when it activates the transcription of FT, which encodes a protein with similarity to Raf kinase inhibitor proteins (Kardailsky et al. 1999; Kobayashi et al. 1999). The activation of FT by CO appears to occur specifically in the phloem, where $C O$ and FT are both expressed (Takada and Goto 2003; An et al. 2004). The biochemical function of FT recently became clearer with the demonstration that it interacts in yeast with a bZIP transcription factor, FD (Abe et al. 2005; Wigge et al. 2005). The FT/FD heterodimer is proposed to activate the expression of three genes encoding MADS-box transcription factors: the floral meristem identity gene APETALA1 (AP1) in floral meristems (Abe et al. 2005; Wigge et al. 2005), and FRUITFULL (FUL) and SEPALLATA3 (SEP3) in the leaf (Teper-Bamnolker and Samach 2005).

A role for FT in activating $A P 1$ in the floral meristem is in contrast to the FT mRNA expression pattern, which appears to be specific to the vascular tissue (Takada and Goto 2003; Abe et al. 2005). Furthermore, misexpression of $C O$ specifically in the phloem complemented a $c O$ mutation, and misexpression of FT in the phloem or the meristem complemented $c o$ and $f t$ mutations (An et al. 2004; Ayre and Turgeon 2004; Abe et al. 2005). Taken together, these observations led to the suggestion that FT may encode a transmissible signal that is transported through the phloem to the meristem from the leaf during the transition to flowering (An et al. 2004; Abe et al. 2005; Wigge et al. 2005). Recently FT mRNA expressed in the leaf from a heat-shock promoter fusion was shown to reach the shoot apex, suggesting that the FT mRNA might comprise at least a part of this signal (Huang et al. 2005).

The photoperiod pathway is therefore activated in the leaves and interpreted in the shoot apical meristem. The SOC1 gene, which encodes a MADS-box transcription factor, is one of the earliest targets of photoperiod in the shoot apical meristem. Mutations in SOC1 cause late flowering, and the gene is expressed in the shoot apical meristem within $24 \mathrm{~h}$ of shifting plants from SDs to LDs (Borner et al. 2000; Samach et al. 2000). However, SOC1 is also expressed in the leaves (Borner et al. 2000; Hepworth et al. 2002). Analysis of seedling RNA demonstrated that SOC1 is activated rapidly by induction of $C O$ overexpression and by overexpression of $F T$ /Samach et al. 2000; Michaels et al. 2005; Moon et al. 2005; Yoo et al. 2005). These observations suggest that SOC1 is activated in seedlings by FT, but the spatial pattern of this expression remains unclear because SOC1 is expressed widely and FT is expressed in the leaves but has effects in the meristem. Similarly, induction of a heat-shock promoter fusion to FT causes SOC1 expression in microdissected shoot apices, but these contained small leaves as well as the shoot apical meristem (Huang et al. 2005).

The vernalization pathway of Arabidopsis promotes flowering in response to extended exposure to low temperature. Winter-annual accessions of Arabidopsis in which vernalization promotes flowering generally carry active alleles at two loci, FRIGIDA (FRI) and FLOWERING LOCUS C (FLC), and FRI activates FLC expression (Michaels and Amasino 1999; Sheldon et al. 1999; Johanson et al. 2000). FLC encodes a MADS-box transcription factor that represses flowering. Overexpression of FLC from a widely expressed viral promoter reduced the abundance of SOC1 and FT mRNAs in seedlings (Hepworth et al. 2002; Michaels et al. 2005). SOC1 transcription is likely repressed by FLC directly binding to a CArG box in the SOC1 promoter (Hepworth et al. 2002). In response to vernalization, the abundance of FLC mRNA and protein is reduced (Michaels and Amasino 1999; Sheldon et al. 1999, 2000), and therefore vernalization promotes flowering by reducing FLC expression. The reduction in FLC expression by vernalization involves chromatin remodeling of FLC that requires the VIN3 protein and stabilization of the inactive state by the VRN2 and VRN1 proteins (Gendall et al. 2001; Levy et al. 2002; Sung and Amasino 2004).

Vernalization is generally believed to be perceived at the shoot apex. Localized cooling of shoot apices, which include young leaf primordia as well as the shoot apical meristem, was sufficient for vernalization of celery, chrysanthemum, and Thlaspi arvense (Curtis and Chang 1930; Schwabe 1954; Metzger 1988). However, these studies cannot distinguish between cold responses at the meristem and young leaves. Further evidence indicates that vernalization can occur in leaves. Young leaves of sugar beet plants were induced to produce the floral stimulus by vernalization, and flowering plants were regenerated from vernalized leaves of Luannari biennis and $T$. arvense but not from nonvernalized leaves (Wellensiek 1964; Metzger 1988; Crosthwaite and Jenkins 1993). Therefore physiological analyses carried out in several species provide a complex picture in which vernalization may affect the competence of the meristem to respond to the floral stimulus and/or the capacity of leaves to produce the stimulus (McDaniel 1985).

Genes with key regulatory roles in flowering-time control have been isolated in Arabidopsis, and these pro- 
vide the opportunity to examine more precisely at the molecular level the spatial control of vernalization response. FLC is expressed widely in the plant including in the shoot apical meristem, the root meristem, and in the leaves (Sheldon et al. 2002; Noh and Amasino 2003; Bastow et al. 2004). However, the tissues in which FLC expression is required to repress flowering have not been described. Here we study the spatial interactions between the photoperiod pathway and vernalization response by misexpressing Arabidopsis flowering genes in specific tissues and studying the effects on floweringtime gene expression by in situ hybridization. We conclude that FLC acts to repress both the expression of systemic flowering signals in the leaf and the response to these signals at the meristem. Vernalization reduces FLC expression in both tissues, rendering the meristem responsive to the systemic signals and allowing the leaves to produce these signals.

\section{Results}

FLC represses flowering when expressed in the phloem or the meristem

Winter-annual varieties of Arabidopsis express FLC, a repressor of flowering, at high levels in the autumn, and vernalization promotes spring flowering by repressing FLC expression. FLC is expressed widely, including in the meristem and leaves (Sheldon et al. 2002; Noh and Amasino 2003; Bastow et al. 2004). A series of promoter fusions to FLC was made in order to drive FLC expression in specific tissues, particularly the phloem and the shoot apical meristem, which have critical roles in floral induction. To test the effect of these fusions on flowering time, they were introduced into Columbia flc-3 mutants carrying an active FRI allele (Materials and Methods). The flowering times of these plants were measured under LDs and SDs (Fig. 1). Expression of FLC specifically in the phloem from the SUC2 or ROLC promoters delayed flowering under LDs, causing the production of at least 25 leaves more than flc-3 mutants (Fig. 1A). FLC expression in the meristem caused a less pronounced effect, but UFO::FLC and KNAT1::FLC plants produced at least 12 leaves more than $f l c-3$ mutants prior to flowering. F1 plants in which both KNAT1::FLC and SUC2::FLC transgenes were combined flowered extremely late under LDs, producing at least 60 leaves more than the latest flowering flc-3 plants; these F1 plants flowered at a similar time to our reference winterannual line FRI FLC (Fig. 1A). In contrast, expression of $F L C$ in the roots from the TobRB7 promoter had no effect on flowering (Fig. 1A). Under SDs expression of FLC only in the meristem or phloem had a weak effect on flowering time, but plants carrying both transgenes were significantly later flowering than $\mathrm{flc}-3$ and flowered at a similar time to FRI FLC (Fig. 1B). These experiments indicate that the floral repressor FLC can act in the meristem and the phloem to delay flowering, and that FLC activity in these two tissues is required for the strong delay of flowering exhibited by nonvernalized, winterannual Arabidopsis.
A
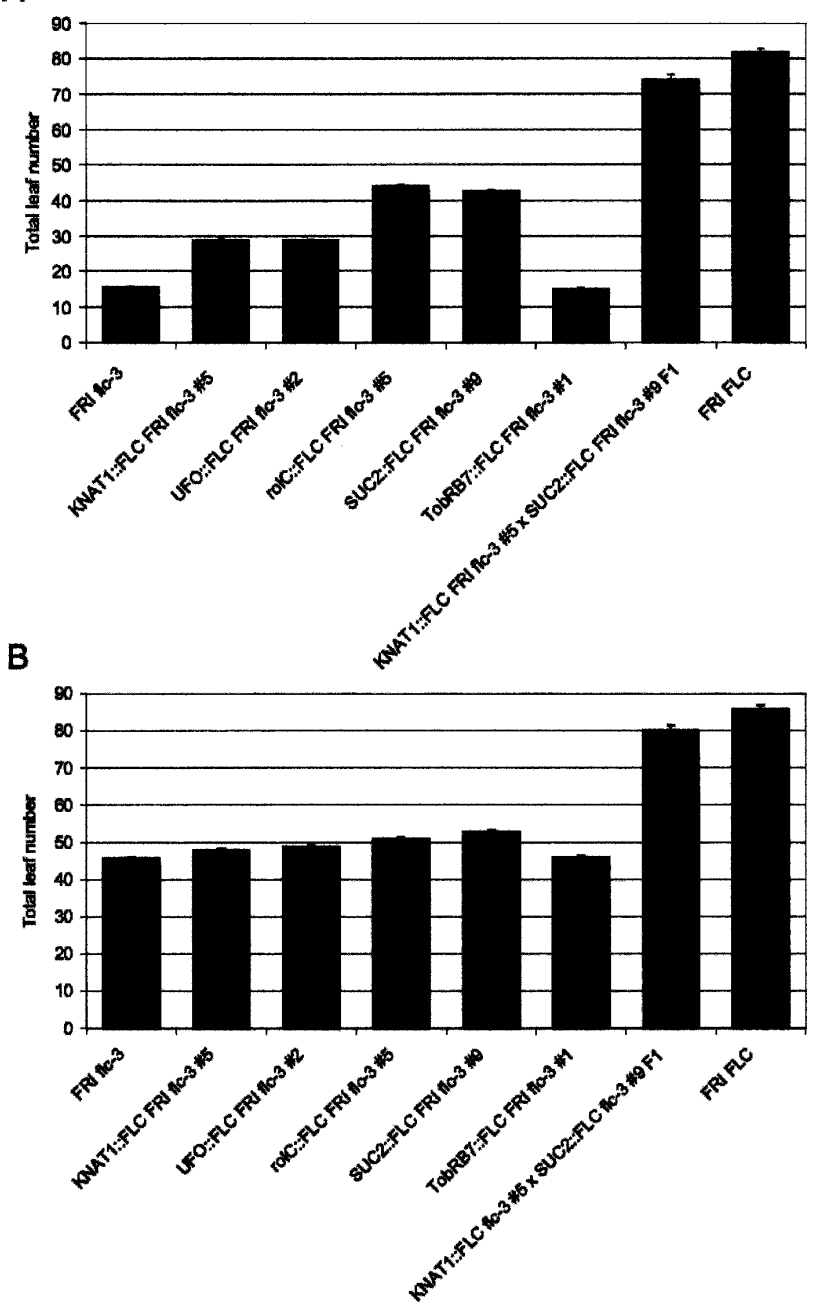

Figure 1. Effect on flowering time of misexpression of $F L C$ from heterologous promoters. (A) Flowering times expressed as total leaf numbers of $f l c-3$ transgenic plants in which $F L C$ is expressed from tissue-specific promoters. All plants were grown under LDs (16 h light $/ 8 \mathrm{~h}$ dark). (B) As $A$, but plants were grown under SDs (10 h light/14 h dark). At least 10 transformants were recovered for each construct, and the flowering time of a representative transformant for each construct is shown.

\section{FLC expression in the phloem reduces SOC1 and FT $m R N A$ levels in the leaf}

The floral integrator genes SOC1 and FT were previously shown to be reduced in expression in whole seedlings of FRI FLC and 35S::FLC plants (Hepworth et al. 2002; Michaels et al. 2005). Whether expression of FLC specifically in the phloem repressed expression of these integrator genes in the leaf was tested by RT-PCR. Abundance of FT and SOC1 mRNAs was strongly reduced in leaves of 10-d-old SUC2::FLC flc-3 plants (Fig. 2). Furthermore, although flowering of KNAT1::FLC flc-3 plants was delayed, FT and SOC1 mRNA levels in the leaves of these plants were not reduced. These data indicate that FLC can act in the phloem to reduce expression of the FT and SOC1 floral integrator genes and that 


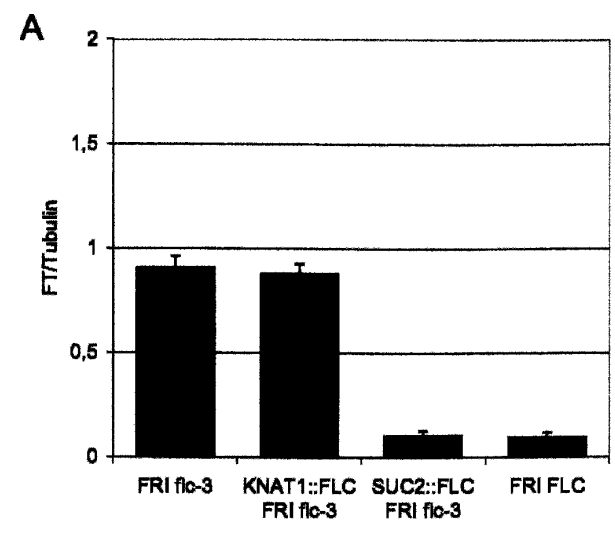

\section{B}

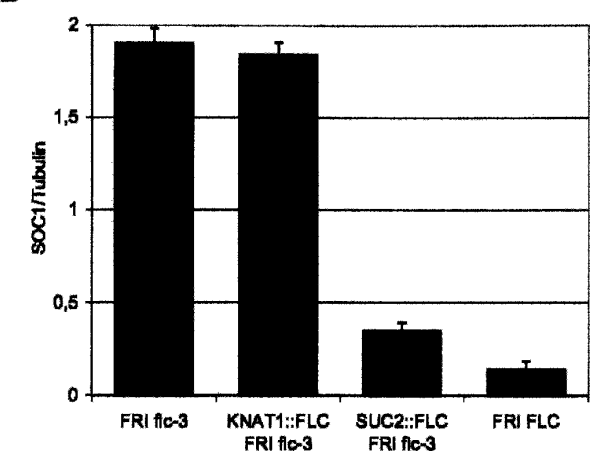

Figure 2. Analysis of mRNA levels in leaves of different genotypes by RT-PCR. (A) FT mRNA abundance in RNA extracted from leaves of $F R I$ flc-3, KNAT1 ::FLC $f 1 c-3$, SUC $2::$ FLC $f l c-3$, and FRI FLC plants grown under LDs. (B) SOC1 mRNA abundance in RNA extracted from leaves of $F R I f 1 c-3$, KNAT1 ::FLC flc-3, SUC2::FLC flc-3, and FRI FLC plants grown under LDs. Leaves were harvested at dusk.

FLC expression in the meristem does not reduce $F T$ or SOC1 expression in leaves.

To determine whether the repression of FT or SOC1 in the leaves was responsible for the late flowering of SUC2::FLC plants, transgenes expressing FT or SOC1 from the SUC2 promoter were introduced into the $S U C 2:: F L C$ background (Fig. 3). Expression of FT in the phloem was previously shown to promote early flowering (An et al. 2004; Abe et al. 2005). SUC2::FT plants were crossed to SUC2::FLC, and the resulting hybrids flowered earlier than wild-type plants under LDs, and with $\sim 30$ leaves fewer than SUC2::FLC plants (Fig. 3C). However, the SUC2::FT SUC2::FLC plants did flower significantly later than SUC2::FT (P $\leq 0.05)$ (Fig. 3C), indicating that FT misexpression did not entirely overcome the effect of FLC. Nevertheless, these data indicate that the effect of FLC expression in the phloem on flowering time is almost entirely overcome by expression of FT in the same tissue from a heterologous promoter.

Whether SOC1 promotes flowering when expressed in the phloem has not been tested. Therefore SOC1 was fused to the SUC2 promoter and introduced into the soc1-1 mutant. The resulting transgenic plants flowered earlier than the soc1-1 mutant progenitor (Fig. 3A,B). For example, SUC2:: SOC1 soc1-1 plants formed $\sim 10$ leaves fewer than soc1-1 mutants under SDs and five leaves fewer under LDs. Therefore, SOC1 is able to promote flowering when specifically expressed in the phloem. However, SUC2::FLC SUC2::SOC1 plants flowered only slightly earlier than SUC2::FLC plants, after forming $\sim 30$ leaves, $\sim 15$ leaves more than SUC2:: SOC1 (Fig. $3 C)$. Therefore increased SOC1 expression in the phloem of SUC2::FLC plants only weakly promotes early flowering. This suggested that SUC2:: SOC1 might promote early flowering by increasing FT expression, because SUC2::FLC delays flowering by repressing FT expression. Consistent with this suggestion, the early flowering of SUC2:: SOC1 plants was completely suppressed by the $f t-7$ mutation (Fig. 3D). Furthermore, FT mRNA levels were much higher in SUC2:: SOC1 plants than in wild type (Fig. 3E), although FT mRNA levels were not reduced in soc1-1 mutants.

Taken together these data indicate that repression of $F T$ is the major mechanism by which FLC expression in the leaf represses flowering and that FLC does not repress events in the leaf that strongly promote flowering downstream of FT.

\section{Alteration of patterns of SOC1 and FD expression in the meristem by FLC activity in the phloem}

FLC expression in the phloem delays flowering, and therefore the effect of FLC activity in the phloem on the induction of flowering gene expression in the meristem was tested. SOC1 expression is one of the earliest available markers for floral induction at the meristem (Borner et al. 2000; Samach et al. 2000). For example, SOC1 mRNA was detected in the meristem by in situ hybridization within $18 \mathrm{~h}$ of transfer of 6 -wk-old Columbia plants from SDs to LDs (Borner et al. 2000). Under our conditions SOC1 mRNA was present in the meristem of 2-wk-old Landsberg erecta plants $16 \mathrm{~h}$ after shifting them from SDs to LDs (Fig. 4A,B), but was not detected $8 \mathrm{~h}$ after this shift (data not shown). The abundance of SOC1 mRNA at the meristem of wild-type Columbia and SUC2::FLC flc-3 plants grown under LDs was then compared by in situ hybridization (Fig. 4). In Columbia, SOC1 mRNA was not detected at the meristem $6 \mathrm{~d}$ after germination, but was present throughout the meristem at $10 \mathrm{~d}$ (Fig. 4C,D). In contrast, in SUC2::FLC flc-3 plants, no SOC1 mRNA was detected at the meristem at either 6 or $10 \mathrm{~d}$ after germination (Fig. 4E,F). These data demonstrate that expression of FLC in the phloem dramatically delays SOC1 expression in the meristem, indicating that FLC expression in the phloem has an indirect repressive effect on SOC1 expression at the meristem.

$F D$ encodes a bZIP transcription factor that promotes flowering and is expressed in the meristem (Abe et al. 2005; Wigge et al. 2005). FD mRNA is present in the meristem of SD-grown plants before floral induction and is increased by shifting plants to LDs (Wigge et al. 2005). The effect of the SUC2::FLC transgene on FD mRNA levels at the meristem was tested and compared with wild-type plants. In the meristem of LD-grown Colum- 
A

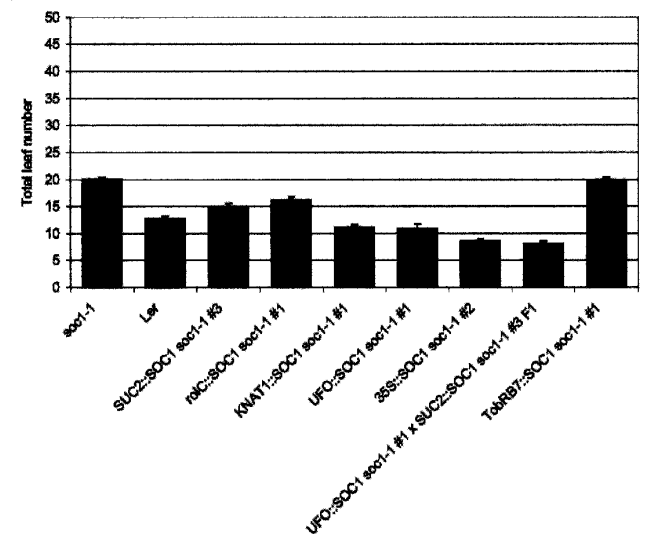

B

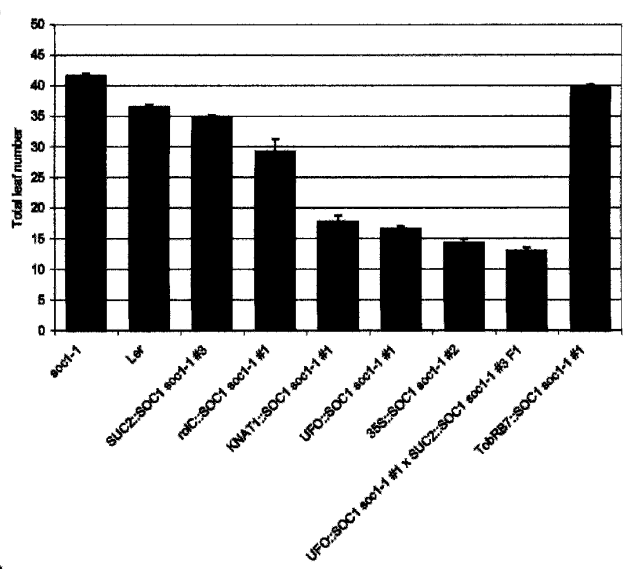

C

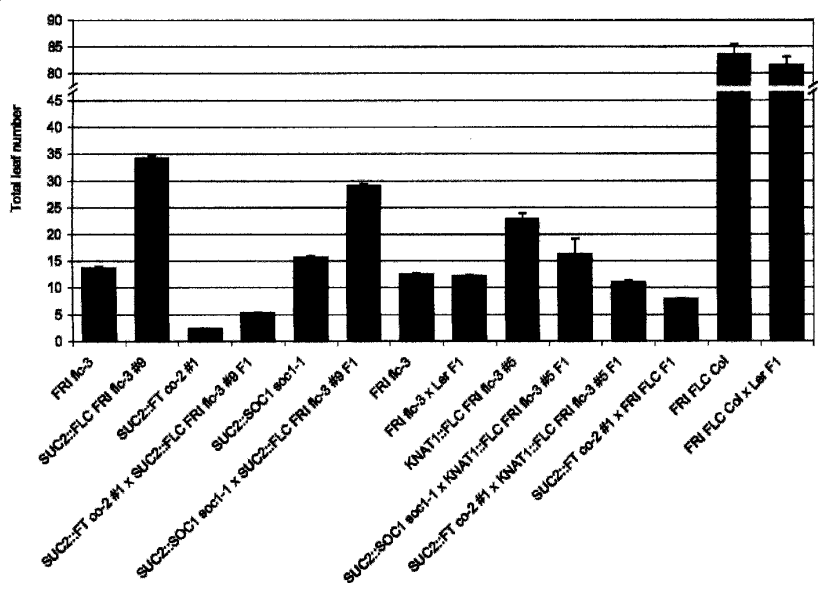

D

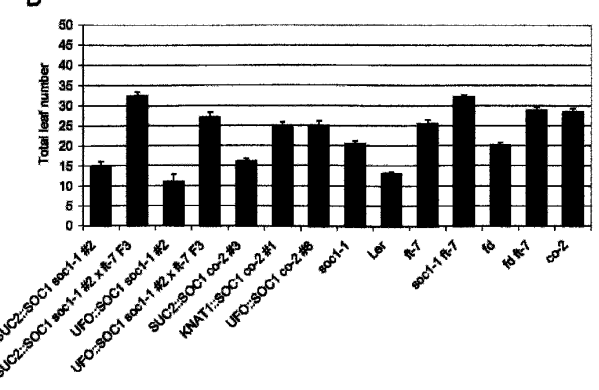

E

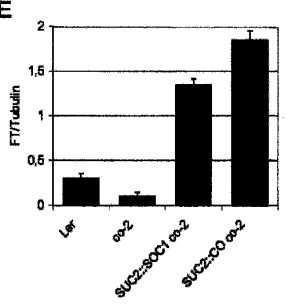

Figure 3. Effects of misexpression of $S O C 1, F T$, and FLC in different genetic backgrounds on flowering time. $(A)$ Flowering times are expressed as total leaf numbers of soc1-1 transgenic plants in which SOC1 is expressed from tissue-specific promoters. All plants were grown under LDs. $(B)$ As $A$, but plants were grown under SDs. $(C)$ Flowering times of transgenic plants misexpressing $F L C$ and either FT or SOC1. Plants were grown under LDs. Some genotypes involve crosses of Columbia FRI flc-3 to Ler, but because the Ler FLC allele does not respond to FRI, no activation of endogenous FLC occurs (Michaels and Amasino 1999). (D) Flowering times of transgenic lines expressing SOC1 from tissue-specific promoters in co-2 or soc1-1 and carrying the $f t-7$ mutation, as well as double mutants carrying $f t-7$ and other flowering-time mutations. Plants were grown under LDs. (E) Analysis of FT mRNA abundance by RT-PCR in the leaves of Ler, co-2, SUC2::SOC1 co-2, and SUC2::CO co-2 grown under SDs. Leaves were harvested at dawn.

bia plants, FD mRNA was detected $6 \mathrm{~d}$ after germination and was strongly increased in abundance 8 and $10 \mathrm{~d}$ after germination as floral induction proceeded (Fig. 5A). In SUC2::FLC plants, FD mRNA was also present at the meristem at similar levels to that detected in wild-type plants $6 \mathrm{~d}$ after germination but remained at this level at 8 and $10 \mathrm{~d}$ after germination (Fig. 5A). This suggests that $F L C$ expression in the phloem does not prevent expres- 

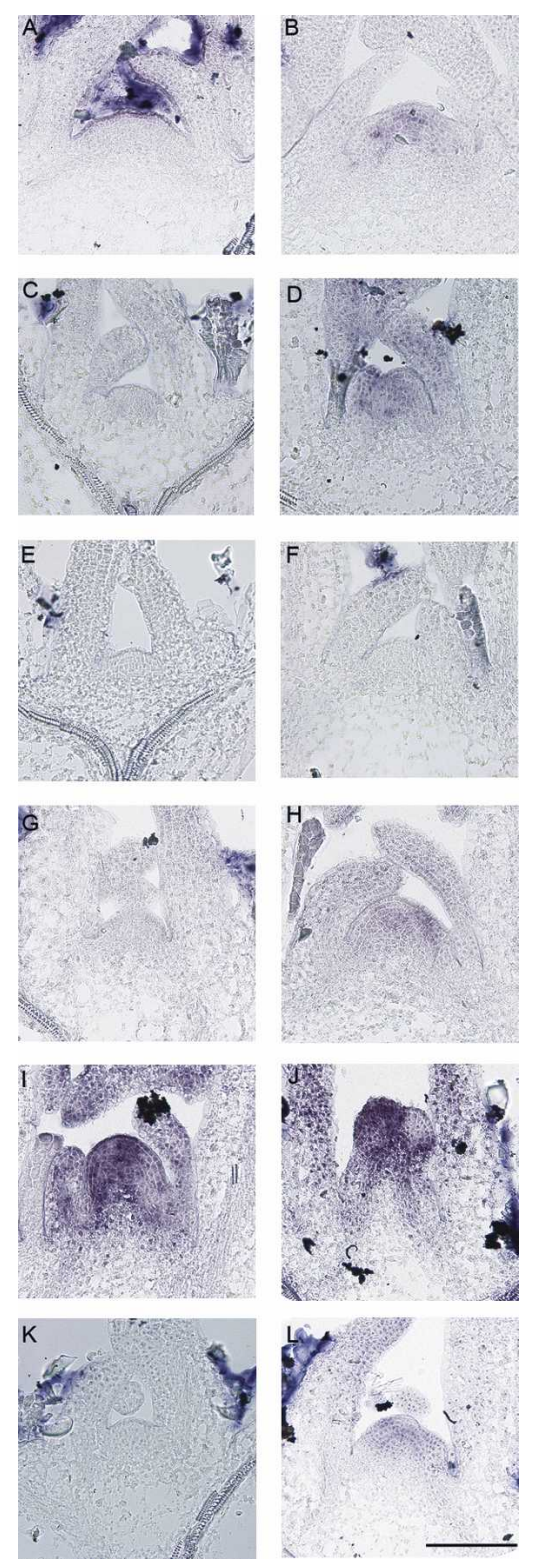

Figure 4. Analysis of SOC1 expression in the meristem by in situ hybridization. (A) Ler plants were grown under 14 SDs $18 \mathrm{~h}$ light) and shifted to LDs (16 h light) at dawn. This plant was harvested $8 \mathrm{~h}$ after dawn, which represented the end of the SD treatment or Time $0($ left $)$. $(B)$ This plant was harvested from the same experiment as $A$ but at dawn the next day, $16 \mathrm{~h}$ after the end of the last SD. SOC1 expression was detected in the meristem. $(C, D)$ Columbia (Col) wild type grown under LDs for $6 \mathrm{~d}$ $(C)$ and $10 \mathrm{~d}(D)$. SOC1 expression is detectable in the 10-d-old plant. $(E, F)$ Meristem of SUC2::FLC flc-3 (Col) plants after 6 LDs $(E)$ and 10 LDs $(F)$. SOC1 expression is not detected. $(G, H)$ $f t-7$ (Ler) plants after 10 LDs $(G)$ and 20 LDs $(H)$. SOC1 expression is only detected weakly after 20 LDs. $(I, J)$ Meristems of Ler $(I)$ and SUC2::CO co-2 $(J)$ after 10 LDs. $(K, L)$ SUC2::CO $f t-7 \mathrm{co}-2$ after $10 \mathrm{LDs}(K)$ and $16 \mathrm{LDs}(L)$. All SUC2::CO co-2 transgenic lines are in a Ler background. Bar, $100 \mu \mathrm{M}$.

sion of $F D$ at the meristem during vegetative growth prior to floral induction, but prevents its up-regulation associated with flowering.
FT and FD promote SOC1 expression at the meristem during floral induction

FLC expression in the phloem reduces FT mRNA levels in the leaf and delays the appearance of SOC1 mRNA in the meristem. Therefore FT activity in the phloem may be required for early expression of SOC1 in the meristem. To test this prediction, the effect of the $\mathrm{ft}-7 \mathrm{mu}-$ tation on SOC1 mRNA levels at the meristem was tested. In $\mathrm{ft}-7$ mutants, SOC1 expression in the meristem was severely delayed so that it was not present $10 \mathrm{~d}$ after germination and only weakly $20 \mathrm{~d}$ after germination (Fig. 4G,H). In contrast, in the meristem of $10-\mathrm{d}$ old Landsberg erecta control plants grown under LDs, SOC1 mRNA was present at high levels (Fig. 4I). Therefore SOC1 expression is delayed in $\mathrm{ft}-7$ mutants by $>10 \mathrm{~d}$. Furthermore, transgenic plants in which $C O$, a positive regulator of FT expression, was expressed in the phloem from the SUC2 promoter caused early flowering through the up-regulation of FT expression in the phloem (An et al. 2004). Therefore, SOC1 mRNA levels were tested in the meristems of SUC2::CO and $f t-7$ SUC2::CO plants. In 10-d-old SUC2::CO plants, SOC1 mRNA was present at the meristem at higher levels than in wild-type plants, and floral primordia were already visible (Fig. 4J). However, the early appearance of SOC1 mRNA at the meristem of SUC2::CO plants is severely delayed by the $\mathrm{ft}-7$ mutation, so that in meristems of 10 -d-old $\mathrm{ft}$-7 SUC2::CO plants SOC1 mRNA was not detected and was only weakly detected in 16-d-old plants (Fig. 4K,L). These results demonstrate that FT is required for early activation of SOC1 in the meristem under LDs and FT function in the phloem increases SOC1 activity in the meristem.

Several observations indicated that FD may be required along with FT to activate SOC1 expression. For example, $F D$ mRNA is present in the meristem before the appearance of SOC1 mRNA (Figs. 4, 5; Wigge et al. 2005), FD interacts with FT to regulate expression of AP1 (Abe et al. 2005; Wigge et al. 2005), and FT is required for SOC1 expression in the meristem (Fig. 4G,H). Therefore, whether FD regulates SOC1was tested by studying the effect of the $f d-1$ mutation on SOC1 mRNA levels in the meristem (Fig. 6B). In $f d-1$ mutants SOC1 mRNA was not detected in the meristem 8,10 , and $12 \mathrm{~d}$ after germination, although wild-type Landsberg erecta control plants showed weak SOC1 expression at $8 \mathrm{~d}$ and much stronger expression at 10 and $12 \mathrm{~d}$ after germination (Fig. 6B). Similarly, 2-wk-old Columbia plants shifted from SDs to LDs showed SOC1 expression in the meristem after $16 \mathrm{~h}$ in LDs, while $f d-3$ plants treated in the same way showed no SOC1 expression after $24 \mathrm{~h}$ in LDs (Fig. 6C). These data indicate that FD is required for early SOC1 expression at the meristem during floral induction.

Taken together, these experiments indicate that FT and FD are required for the induction of SOC1 expression at the shoot apical meristem, which is one of the earliest defined steps in floral induction. 
Searle et al.

Figure 5. Analysis of $F D$ and $F L C$ expression in the meristem by in situ hybridization and RT-PCR. $(A) F D$ expression in the meristem of Col wild-type, KNAT1 ::FLC $f l c-3$, and SUC2::FLC flc-3 plants after 6 LDs (left column), 8 LDs (middle column), and 10 LDs (right column). FLC transgenic lines are in a Col background. Bar, $100 \mu \mathrm{M} .(B)$ Analysis of $F D$ mRNA level by RTPCR in FRI flc-3, KNAT1::FLC flc-3, FRI FLC plants after 10 LDs. (C) FD expression in the meristem of $\mathrm{ft}$ mutants after 6 LDs (left), 8 LDs (middle), and 10 LDs (right). (D) Before vernalization, FRI FLC plants were grown under 14 SDs (top left), then vernalized for $28 \mathrm{~d}$, and immediately after vernalization (top right), FLC expression was analyzed in the meristem by in situ hybridization. FD expression before vernalization (bottom left) and after vernalization (bottom right) of the same plants.
A
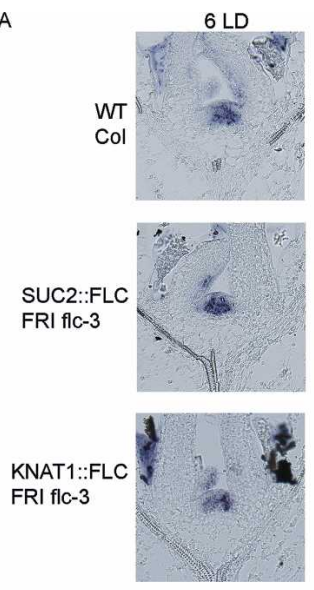

c
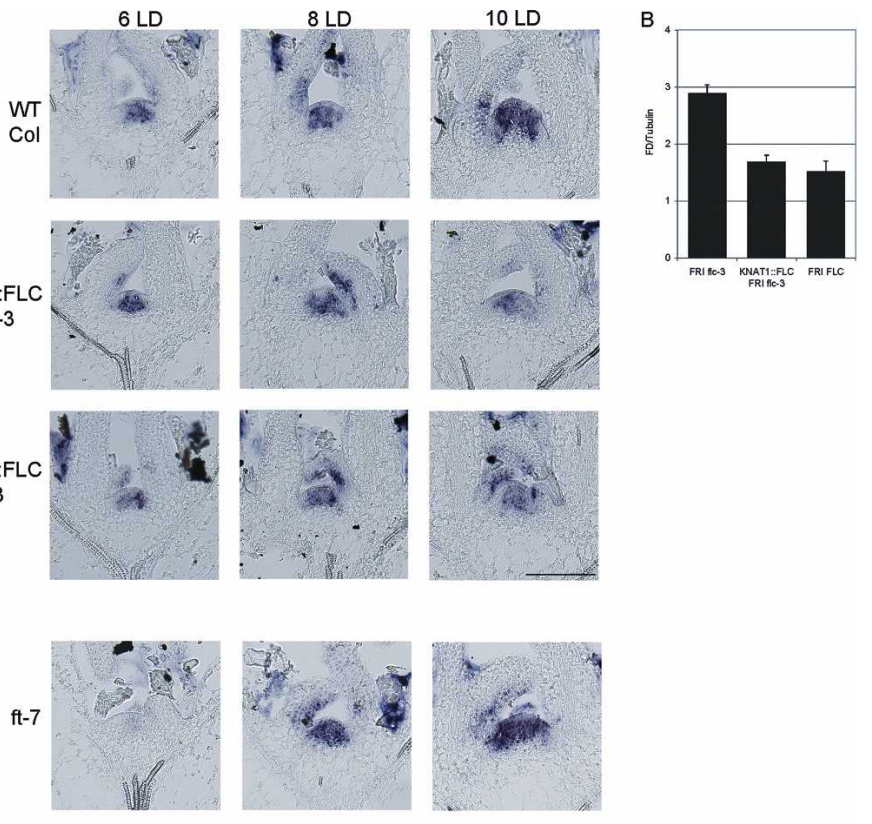

D

E

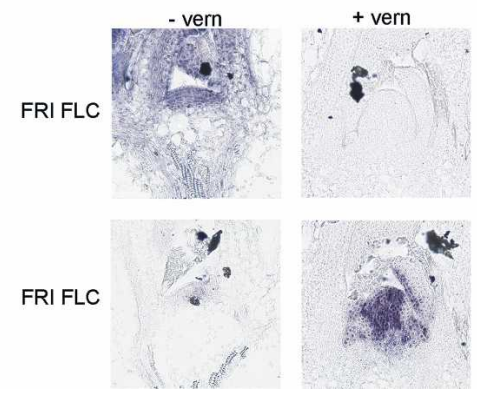

FT activity is not required for increased FD expression in the meristem during floral induction

Expression of FLC in the phloem reduced FT mRNA levels in the leaf (Fig. 2) and $F D$ mRNA levels in the meristem (Fig. 5A). This suggested that FT activity in the leaf might be required for the increased expression of $F D$ that occurs during the transition to flowering. $F D$ mRNA was therefore compared by in situ hybridization in $f t-7$ mutants, $S U C 2:: F L C$, and wild-type plants grown under LDs (Fig. 5A,C). As previously described, in wildtype plants $F D$ expression rises between 6 and $10 \mathrm{~d}$ after germination, whereas in SUC2::FLC plants, FD mRNA remains at the low levels detected at $6 \mathrm{~d}$. The $f t-7$ mutant showed the same pattern of $F D$ expression as wildtype plants, so that the abundance of $F D$ mRNA rose between 6 and $10 \mathrm{~d}$ and at $10 \mathrm{~d}$ was similar to that of wild-type plants. Therefore, although $\mathrm{ft}-7$ mutants are severely late flowering they are not delayed in the rise in $F D$ expression that occurs between 6 and $10 \mathrm{~d}$ under LDs. Furthermore, the reduction of $F D$ expression at the meristem observed in $S U C 2:: F L C$ plants suggests that FLC represses expression of other genes in the phloem that are required for the up-regulation of $F D$ at the meristem.
FLC activity in the meristem represses SOC1 and delays FD expression

$F L C$ expression in the meristem delayed flowering but did not reduce FT mRNA levels in the leaf (Figs. 1, 2). Therefore, the effect of FLC on the expression of flowering-time genes in the meristem was tested by in situ hybridizations performed on KNAT1::FLC plants.

SOC1 expression in the meristem of KNAT1::FLC plants was tested by in situ hybridization, because FLC represses SOC1 in leaves (Fig. 2B). In Columbia plants grown under LDs, SOC1 expression was not detected in the meristem $6 \mathrm{~d}$ after germination, but was present strongly $10 \mathrm{~d}$ after germination (Fig. 6A). In contrast, in KNAT1::FLC plants, SOC1 mRNA was not present $10 \mathrm{~d}$ after germination. This analysis suggests that FLC expression in the meristem delays and reduces the expression of SOC1.

The flowering-time gene $F D$ is expressed in the meristem prior to floral induction, and is required along with FT for the up-regulation of SOC1 (Fig. 6B,C; Abe et al. 2005; Wigge et al. 2005). Therefore, whether FLC represses SOC1 expression at least in part by reducing $F D$ expression in the meristem was tested. $F D$ expression was compared in Columbia and KNAT1::FLC plants by 
A

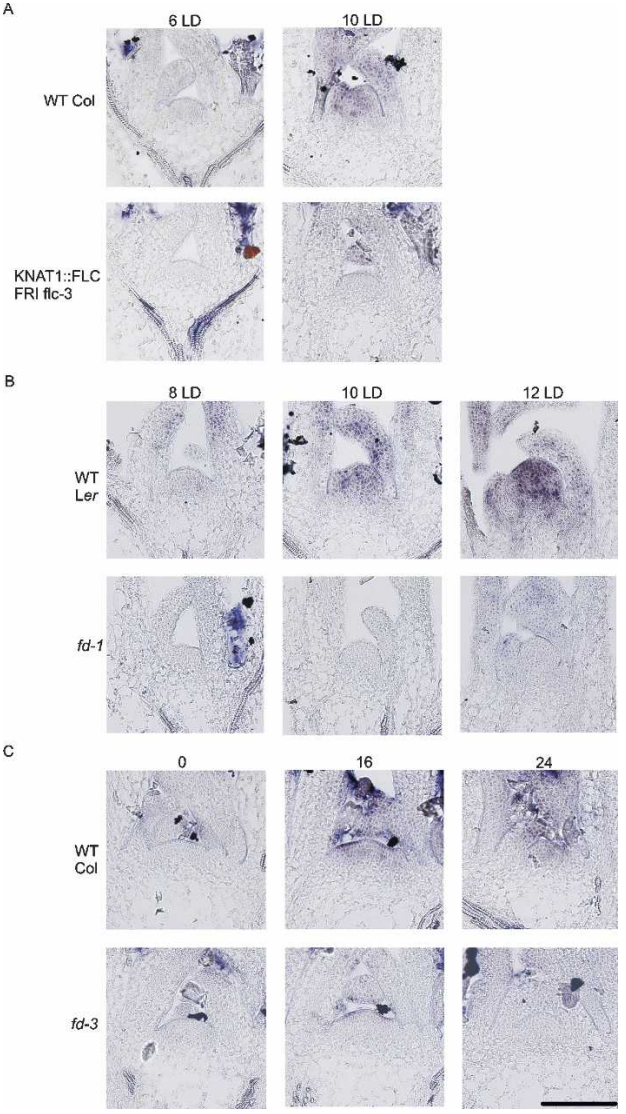

Figure 6. Analysis of SOC1 expression in the meristem by in situ hybridization. (A) SOC1 expression in the meristem of wild-type Col and KNAT1 : FLC flc-3 plants grown for 6 and 10 LDs. SOC1 expression is detectable in wild-type (WT) but not KNAT1::FLC after 10 d. (B) SOC1 expression in the meristem of Ler and $f d-1$ (Ler) after 8, 10, and 12 LDs. $(C)$ Columbia and $f d-3$ (Col) mutant plants were grown under SDs (for $14 \mathrm{~d}$ ) and shifted to LDs at dawn. Eight hours after dawn was the end of the SD treatment and was designated as the zero time point $(0$, left). Sixteen hours later, SOC1 expression was detected in the meristem of Col but not the $f d-3$ mutant $(16$, middle). Twentyfour hours latter, SOC1 expression was still not detected in the fd-3 mutant (24, right). Bar, $100 \mu \mathrm{M}$.

in situ hybridization (Fig. 5A). In KNAT1::FLC plants, $F D$ expression in the meristem was similar to that in Columbia plants $6 \mathrm{~d}$ after germination, but did not increase at 8 and $10 \mathrm{~d}$, continuing to be expressed weakly (Fig. 5A). This analysis suggests that FLC expression in the meristem does not affect the early appearance of $F D$ mRNA in the meristem, but delays the increase in $F D$ expression associated with floral induction, as was shown earlier for FLC expression in the phloem.

Expression of SOC1 in the meristem promotes early flowering

SOC1 is expressed in the leaf and meristem (Borner et al. 2000; Samach et al. 2000), and its expression in the leaf accelerates flowering (Fig. 3). In addition, SOC1 mRNA levels in the meristem are increased by FT and FD and reduced by FLC (Figs. 4, 6). This suggests that expression of SOC1 in the meristem is closely associated with the initiation of flowering. To test whether SOC1 expression in the meristem is sufficient to complement the soc1-1 mutation, SOC1 was expressed from the KNAT1 and UFO promoters in the Soc1-1 mutant. KNAT1::SOC1 soc1-1 and UFO::SOC1 soc1-1 transgenic plants flowered after forming 10 fewer leaves than soc1-1 mutants under LDs, and $\sim 20$ fewer leaves under SDs (Fig. 2A,B). The KNAT1:: SOC1 soc1-1 and UFO::SOC1 soc1-1 transgenic plants were also significantly earlier flowering than wild type, particularly under SDs (Fig. 2). In contrast, expression of SOC1 in roots from the TobRB 7 promoter had no effect on flowering time (Fig. 2). These data indicate that expression of SOC1 in the meristem causes a pronounced early-flowering phenotype that is more severe than the effect of expressing SOC1 in the phloem in soc1-1 mutants.

To test whether the functions of SOC1 in the phloem and meristem have additive effects on flowering, UFO : : SOC1 SOc1-1 and SUC2:: SOC1 soc1-1 transgenic plants were crossed and the flowering times of the resulting F1 plants measured under LDs and SDs (Fig. 1). The F1 plants flowered earlier than either progenitor, after forming 30 leaves fewer than soc1-1 under SDs and 12 leaves fewer than soc1-1 under LDs. The UFO:: SOC1 SUC2:: SOC1 soc1-1 plants flowered with a similar number of leaves to $35 S:$ SOC1 soc1-1 plants, in which SOC1 expression occurs at high levels in most tissues. Taken together, these data indicate that SOC1 promotes flowering from the meristem as well as the phloem, and suggest that the reduction in SOC1 mRNA abundance detected in the meristem of KNAT1::FLC and $f t-7$ plants contributes to the delay in flowering time of these plants.

SOC1 expression is activated by FT and FD and is downstream of the systemic signal induced in the leaves (Figs. 4, 6). Whether SOC1 expression at the meristem is sufficient to induce flowering in plants impaired in the production of the systemic signal in the leaves was tested by expressing SOC1 specifically in the meristem of $c O-2$ and $f t-7$ mutants. Expression of SOC1 from the UFO::SOC1 or KNAT1::SOC1 transgenes in the co-2 mutant caused earlier flowering, accelerating flowering by around five leaves (Fig. 3D). Similarly UFO::SOC1 soc1-1 ft-7 plants flowered with around seven leaves fewer than soc1-1 ft-7 (Fig. 3D). These data indicate that SOC1 expression at the meristem is sufficient to weakly promote flowering independently of the $C O$ and FT genes that are expressed in the phloem. However, expression of SOC1 in the meristem is not sufficient to overcome the effect of $\mathrm{co}-2$ or $\mathrm{ft}-7$ mutations on flowering time, suggesting that the FT-dependent signal produced in the leaf must have additional targets in the meristem.

\section{Flowering-time gene expression in FRI FLC plants}

The effects of FLC expression in specific tissues on the activity of flowering-time genes was tested in the experi- 
ments described above. To confirm the significance of these observations for plants expressing FLC from its own promoter, expression of FLC, SOC1, FT, and FD was tested by RT-PCR or in situ hybridization in vernalized and nonvernalized winter-annual FRI FLC plants.

FT and SOC1 mRNA levels were tested by RT-PCR in RNA extracted from leaves of FRI FLC and flc-3 plants. The abundance of SOC1 and FT mRNA was reduced in leaves of FRI FLC plants, as previously described for SUC2::FLC plants (Fig. 2). This result indicates that FLC represses SOC1 and FT in the leaves in winter annuals.

FLC expression specifically in the meristem delayed flowering and altered SOC1 and FD expression. In FRI FLC plants, the expression of FLC at the shoot apex was previously described by staining whole seedlings carrying FLC::GUS transgenes (Sheldon et al. 2002; Noh and Amasino 2003; Sung and Amasino 2004). To confirm the presence of FLC mRNA at the shoot apical meristem in FRI FLC plants, in situ hybridizations were performed. In 14-d-old FRI FLC plants grown under SDs and not exposed to vernalization, FLC mRNA was detected in the meristem and young leaf primordia (Fig. 5D). In contrast, no FLC mRNA was detected in the meristem or young leaf primordia of plants exposed to low temperatures for $28 \mathrm{~d}$ (Fig. 5D). This result demonstrates that FLC expression in the shoot meristem is repressed by vernalization.

FD mRNA was detected at low level in the meristem of KNAT1::FLC plants similar to that of wild-type plants prior to floral induction. RT-PCR analyses indicated that shoot apices of FRI FLC plants contained lower levels of FD mRNA than plants lacking active FRI alleles (Fig. 5B). Furthermore, in situ hybridization showed that in the shoot meristems of FRI FLC plants, $F D$ mRNA was present at low levels before vernalization (Fig. 5D). In contrast, after vernalization of FRI FLC plants, $F D$ was expressed strongly in the meristem (Fig. 5D).

These experiments demonstrate that in FRI FLC plants, in which FLC is expressed widely, the effects of FLC on SOC1, FT, and FD mRNA levels is consistent with those observed in transgenic plants expressing FLC in specific tissues.

\section{Additive effects of FLC in the leaf and meristem}

As discussed above, crossing SUC2::FLC and KNAT1::FLC plants, which express FLC specifically in the phloem or the meristem, respectively, produced progeny that flowered much later than either parent. The molecular analysis presented above suggests that this additive effect is due to reducing both expression of genes such as FT in the leaf and more strongly delaying expression of genes such as SOC1 and FD in the meristem. To test this additive effect, the flowering times of the $f t-7$ $f d-1$ and $f t-7$ soc1-1 double mutants (Fig. 3D), which partly recreate the effects of FLC in both the leaf and meristem, were scored. The $f t-7 f d-1$ and $f t-7$ soc1-1 double mutants flowered later than either of the progenitor single mutants, indicating that these mutations have additive effects on flowering time. This is consistent with the severe delay in flowering observed in FRI FLC plants being due to the combined effect of repressing expression of several flowering-time genes, including FT expression in the leaf, as well as FD and SOC1 expression at the meristem.

\section{FLC binds directly to FT, SOC1, and FD in vivo}

FLC reduces expression of SOC1, FT, and FD (Figs. 2, 3E, $4,5,6)$. FLC was previously shown to bind in vitro to a CArG box in the SOC1 promoter (Hepworth et al. 2002). Chromatin immunoprecipitation (ChIP) was used to test binding of FLC to the FT and SOC1 promoters in vivo. Transgenic plants were constructed expressing an FLC:HA fusion protein from the CaMV $35 S$ promoter. One line that flowered later than wild-type plants was used for the ChIP experiments (Materials and Methods). Proteins were cross-linked to the DNA of these plants and precipitated using an anti-HA antibody. PCR was then performed to test for fragments of the FT or SOC1 genes in the precipitated DNA (Fig. 7A,B). The region of the SOC1 promoter previously shown to bind the FLC protein in vitro was efficiently precipitated, consistent with FLC:HA binding to this site in vivo. Similarly, in the FT gene a region within the first intron of FT was precipitated efficiently (Fig. 7B). To position the site of FLC:HA binding within the FT gene more precisely, PCR was performed on five DNA fragments spanning the first exon and the first and second introns of FT. The efficiency of precipitation was determined for each fragment, and this produced a bell-shaped curve with a peak near the $5^{\prime}$ end of the first intron (Fig. 7C). This region contains a CArG-box consensus sequence consistent with FLC:HA binding.

Finally, binding of the FLC:HA protein to the FD promoter region was tested. A total of four fragments extending from ATG to 4316 base pairs (bp) were tested. One fragment was immunoprecipitated at high efficiency, and this extended from 4316 to $3500 \mathrm{bp}$. This region contains a CArG-box consensus sequence, suggesting that FLC regulates FD expression by binding directly to the promoter region.

The ChIP experiments therefore suggest that FLC represses FT, SOC1, and FD expression prior to vernalization at least partly by binding directly to CArG boxes within the first intron of FT and the promoter regions of SOC1 and FD.

\section{Discussion}

We have shown that the vernalization response in Arabidopsis requires derepression of key regulatory genes in the leaf and meristem (Fig. 8). Prior to vernalization, repression of FT in the leaf by the FLC transcription factor prevents formation of a systemic signal and thereby indirectly delays expression of the flowering-time gene SOC1 at the meristem. FLC activity in the leaf also delays expression of $F D$ at the meristem, and, based on the 
A

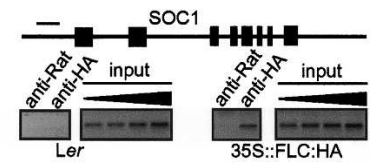

B

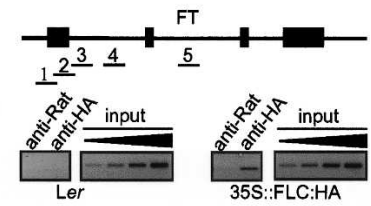

C

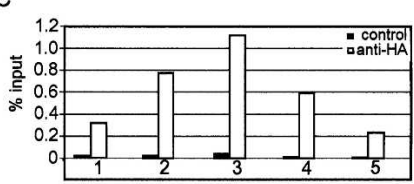

D

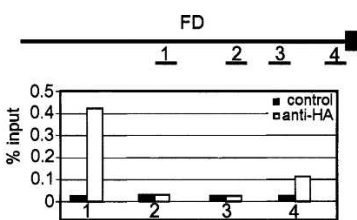

Figure 7. ChIP analysis of SOC1, FT, and FD loci. (A) A SOC1 promoter fragment containing the FLC-responsive CArG box was amplified from DNA immunoprecipitated with control (anti-Rat IgG) or anti-HA antibodies from Ler or 35S ::FLC:HA Ler. A twofold dilution series of the input DNA was amplified as a semiquantitative standard. The SOC1 promoter region assayed is indicated by a thin horizontal line. Coding regions are indicated by shaded boxes. (B) Five FT fragments spanning introns 1 and 2 were amplified from immunoprecipitated DNA as described in $A$. Fragment 3 contains a putative FLC-responsive CArG box and was amplified from immunoprecipitated DNA, and twofold dilutions of the input DNA are shown as a control. Horizontal lines numbered 1-5 indicate assayed fragments. (C) Quantification of the amplified signal from fragments 1-5 relative to the input. $(D)$ Four fragments spanning the FD promoter region were amplified from immunoprecipitated DNA as described in $A$.

analysis of $f t$ mutants, this occurs independently of the repression of FT expression by FLC. Activity of FLC specifically in the meristem did not affect $F T$ expression in the leaf but reduced SOC1 mRNA levels and prevented the up-regulation of $F D$ associated with flowering. Therefore activity of FLC in the meristem suppresses the response to the FT signal formed in the leaves by directly repressing SOC1 and $F D$, which encodes an FT-interacting protein. These data suggest that flowering is delayed prior to vernalization because FLC represses production of at least two systemic signals in the leaves, one that requires FT and a second that appears to be independent of FT, and impairs the competence of the meristem to flower in response to these signals through the repression of FD and SOC1. The delays in flowering caused by FLC in the leaf and meristem are additive, so that expression of FLC in both tissues causes a much more severe delay in flowering. In winter-annual FRI FLC plants, vernalization reduces $F L C$ expression in the young leaves and the meristem. We conclude that vernalization acts in Arabidopsis both to allow production of floral stimuli in the leaves and response to these stimuli in the meristem.

\section{Repression of flowering by FLC expression in phloem} companion cells

$F L C$ expression in the phloem companion cells from the $S U C 2$ or ROLC promoters delays flowering. Previously, $\mathrm{CO}$, a photoperiod pathway component, was also shown to act in the phloem companion cells to promote flowering, and to do so through the cell-autonomous activation of FT transcription (An et al. 2004; Ayre and Turgeon 2004). Expression of $F T$ in the companion cells also complements either co or $f t$ mutations, and FT is expressed in the phloem in wild-type plants (Takada and Goto 2003; An et al. 2004; Abe et al. 2005). Therefore, the antagonism between the photoperiod pathway and FLC-mediated repression of flowering that was previously described for plants overexpressing these genes in all tissues from the $35 \mathrm{~S}$ promoter probably occurs in the phloem companion cells. This conclusion is strengthened by our observation that expression of FLC in the

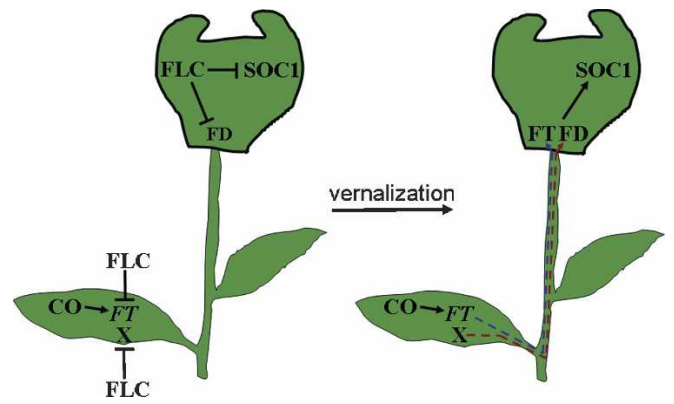

Figure 8. A schematic diagram illustrating the interactions between FLC and the photoperiod pathway in the leaf and meristem and the effect of vernalization on these interactions. Prior to vernalization (diagram on left), FLC acts in the leaf to repress transcription of $F T$ and of other genes that are illustrated as X. In the meristem, FLC represses transcription of $F D$ and of SOC1. Vernalization reduces FLC expression both in the leaf and the meristem. Removal of FLC from the leaves causes FT and $X$ to be expressed. FT controls a systemic signal (shown in blue) that is required for SOC1 expression at the meristem. FT mRNA was recently proposed to move from the leaves to the meristem during floral induction (Huang et al. 2005), and therefore this signal might be comprised of FT mRNA. FD is also required at the meristem for SOC1 expression, and because FT and FD interact (Abe et al. 2005; Wigge et al. 2005), this heterodimer might activate SOC1 expression directly or indirectly. $F D$ expression at the meristem also rises during floral induction. The increase in $F D$ expression does not require FT but is blocked by expression of FLC in the leaves. Therefore we propose that in the leaves, FLC blocks expression of X, which controls a second systemic signal (shown in red) that increases $F D$ expression at the meristem. The reduction of FLC expression in the meristem during vernalization allows FD and SOC1 expression in the meristem to rise in response to the $\mathrm{X}$ signal and FT/FD, respectively. 
companion cells and the presence of dominant FRI FLC alleles in winter annuals strongly reduces SOC1 and FT mRNA levels, as shown previously in total RNA of 35S::FLC plants (Hepworth et al. 2002).

FLC activity in the leaf probably delays flowering by repressing formation of systemic signals and thereby indirectly reducing the activation of genes in the meristem (Fig. 8). The reduction of FT expression by SUC2::FLC likely contributes to repression of systemic signaling based on previous reports of the function of FT (An et al. 2004; Abe et al. 2005; Huang et al. 2005; Wigge et al. 2005). Consistent with this idea, SOC1 expression was reduced in the meristem in $f t-7$ mutants or in SUC2::CO ft-7 plants as well as in SUC2::FLC plants. SOC1 expression in the meristem is one of the earliest indications of floral induction, occurring within $16 \mathrm{~h}$ of the transfer of plants from SDs to LDs. The reduction in SOC1 expression in the meristem of $\mathrm{ft}-7$ mutants demonstrates that activation of FT in the phloem is required for induction of one of the earliest events of floral evocation in the meristem. This conclusion is consistent with the observation that induction of FT overexpression in a single leaf led to activation of SOC1 in microdissected shoot apices, although whether this increase in SOC1 mRNA levels occurred in leaf primordia, vascular tissue, or the shoot meristem was not determined (Huang et al. 2005).

Reduction of FT expression by FLC in the phloem may also prevent gene activation in the leaf. Consistent with this idea, expression of the SEP3 and FUL genes was recently shown to be increased in plants overexpressing FT from the $35 \mathrm{~S}$ promoter and to be reduced in $\mathrm{ft}$ mutants compared with wild-type plants (Teper-Bamnolker and Samach 2005). However, these genes are unlikely to represent further major targets for FLC in the leaf because $S U C 2:: F T$ could largely overcome the effect of expressing $F L C$ in the leaf.

Expression of FLC in the phloem also reduced expression of $F D$ at the meristem. Unlike the effect of SUC2::FLC on SOC1 expression at the meristem, the effect on $F D$ cannot be explained by repression of $F T$ expression because in $\mathrm{ft}$ - 7 mutants the rise in $F D$ expression was similar to that in wild-type plants. This suggests that the increase in FD mRNA levels observed at the meristem during the transition to flowering is controlled by a phloem signal that is repressed by FLC but is independent of FT. Expression of FLC in the meristem also represses FD expression, so the effect of SUC2::FLC on $F D$ expression might be explained by movement of the FLC mRNA or protein from the phloem of SUC2::FLC plants to the meristem. However, this seems unlikely, because the MADS-box protein AP1 was shown previously not to move between cells (Wu et al. 2003), and we show here that meristem expression of SOC1, a MADS-box protein, has different effects on flowering time than expression of SOC1 in the phloem. Therefore the contrasting effects of SUC2::FLC and $f t-7$ on $F D$ expression support the idea that the floral stimulus contains at least two activities, one that is FT-dependent and regulates SOC1 and a second that is indepen- dent of FT and regulates FD. The importance of the FTindependent signal in the timing of the floral transition is still not clear, and any effect it might have on flowering time can be largely overcome by expressing FT at high level, since SUC2::FT SUC2::FLC plants flowered only slightly later than SUC2::FT plants. Further genetic analysis is required to characterize this signal and its relationship to previously described flowering-time genes.

The significance of SOC1 expression in the leaves has not been clear, and may be related to the recent observation that other MADS-box transcription factors with roles in flowering such as FUL and SEP3 were also shown to be expressed in the leaves and to be regulated by FT (Teper-Bamnolker and Samach 2005). We demonstrated that expression of SOC1 from the SUC2 or ROLC promoters is sufficient to accelerate flowering in a soc1-1 mutant, indicating that SOC1 expression in these tissues can influence flowering. In SUC2:: SOC1 plants, FT expression is increased and $f t-7$ mutations suppress the early flowering of SUC2::SOC1 plants. Therefore, in these plants SOC1 promotes flowering by activating FT expression, and this activation of $F T$ is also likely to be the basis of the strong suppression of the co-2 phenotype by SUC2:: SOC1. However, no reduction in FT expression was observed in soc1-1 mutant seedlings. Furthermore, no increase in FT expression was observed previously when SOC1 was expressed from the $35 S$ promoter (Lee et al. 2000; Yoo et al. 2005). This indicates that the effect of SOC1 on FT expression may only occur when SOC1 is expressed at high levels in the phloem from the SUC2 promoter or that it reflects regulation of FT by SOC1 at other stages of plant development. Alternatively, a reduction in FT expression might not be observed in soc1-1 mutant seedlings due to redundancy with other related MADS-box proteins expressed in the leaf.

The mechanism of FLC-mediated repression of FT and SOC1 in the leaf likely involves direct binding of FLC to the sequences in the first intron of FT and to the promoter region of SOC1. In vivo ChIP studies detected binding of FLC:HA to these regions, and they also contain consensus CArG boxes. The significance of the intron CArG sequence for FT regulation remains to be tested, but these experiments are consistent with previous observations that FLC binds to the SOC1 promoter in vitro and that removal of the CArG-binding site reduces repression of SOC1 expression by FLC (Hepworth et al. 2002).

Repression of flowering by FLC expression in the shoot apical meristem

FLC expression in the meristem reduces expression of the flowering-time genes $F D$ and SOC1. FD encodes a bZIP transcription factor that is expressed in the meristem prior to the onset of flowering, and the abundance of $F D$ mRNA is increased during the transition to flowering (Abe et al. 2005; Wigge et al. 2005). FD protein interacts with FT, and the resulting complex is proposed to activate transcription of the floral meristem identity 
gene AP1 in the floral meristem (Abe et al. 2005; Wigge et al. 2005). Mutations in FD cause late flowering, and we show that $f d$ mutants exhibit reduced and delayed expression of SOC1 mRNA in the meristem of plants grown under LDs or plants shifted from SDs to LDs. Therefore, both FD and FT are required for SOC1 activation in the meristem, suggesting that interaction of these proteins is required for SOC1 expression prior to the activation of AP1 (Abe et al. 2005; Wigge et al. 2005). This extends the role of FT and FD to the earliest known stages in floral induction at the meristem. These data suggest that $F D$ expression confers on the meristem the competence to respond to the FT signal by facilitating the activation of SOC1 by FT. This would represent an earlier stage in meristem competence than the homeobox genes PENNYWISE and POUND-FOOLISH because mutations in these genes impaired competence of the meristem to respond to inductive signals but did not prevent expression of SOC1 (Smith et al. 2004).

FLC expression in the meristem does not affect the initial appearance of $F D$ mRNA, which is expressed at similarly low levels in wild-type and KNAT1::FLC plants, but delays its up-regulation. ChIP experiments indicated that FLC:HA binds directly to a CArG box in the 5' region of FD. Nevertheless, the effect of FLC:HA on $F D$ expression is different from that observed for SOC1 and FT, because a low level of expression of $F D$ was detected in the presence of FLC but FD up-regulation was blocked. This effect may be due to the distal location of the FLC-binding site in the FD promoter. Further experiments are required to test the significance of this FLC-HA-binding site for FD regulation.

Consistent with the previous observation that $F D$ is expressed under SDs prior to flowering (Wigge et al. 2005), our data indicate that $F D$ expression can be divided into two phases, one that occurs prior to floral induction and leads to low-level expression at the meristem that can be maintained through vegetative growth and a second phase that involves up-regulation coincident in wild-type plants with the transition to flowering. However, the up-regulation of $F D$ can be separated from the transition to flowering in $f t-7$ mutants, where it occurs at the same time as in wild-type plants although flowering of $f t-7$ is severely delayed. FLC expression in the meristem maintains FD mRNA levels at the lower level associated with vegetatively growing wild-type plants prior to flowering.

Flowering of plants expressing FLC in the meristem is delayed even although they express FT in the leaf and $F D$ at the meristem at similar levels to wild-type plants when these plants initiate flowering. This suggests that plants expressing FLC in the meristem cannot respond to the FT/FD signal. At least in part this may be due to direct repression of SOC1, which is activated by FT/FD in the meristem, and the appearance of SOC1 mRNA in the meristem is severely delayed by expression of FLC in the meristem. SOC1 may have a major role downstream of FT/FD in the promotion of flowering. In support of this suggestion KNAT1::SOC1 plants promoted earlier flowering of $\mathrm{ft}-7$ mutants, although only weakly. The relatively weak promotion of flowering caused by KNAT1::SOC1 suggests that FT/FD has additional targets in the meristem that are required for early flowering. In FRI FLC plants, vernalization restores the competence of the meristem to respond to FT by reducing FLC expression, allowing FD and SOC1 expression to rise during floral induction.

Our data demonstrate that FLC acts at different levels in the regulatory hierarchy (Fig. 8). FT expression is repressed in the leaf, the up-regulation of $F D$ in the meristem is prevented, and SOC1, a likely target of FT/FD function in the meristem, is repressed by direct binding of FLC. Repression of multiple steps in the hierarchy probably results in much later flowering than would be possible by repressing a single step. This is supported by double-mutant analysis. We found that the $f d-1 \mathrm{ft}-7$ double mutants flowered later than either single mutant, as suggested for $f d-1$ and $f t-3$ alleles, although this was not shown in direct comparisons in the same experiment (Abe et al. 2005). Similarly, the soc1-1 ft-7 double mutant flowered later than either single mutant, as previously shown in the 35S::CO background (Onouchi et al. 2000). Therefore, repression of multiple steps in the flowering hierarchy by FLC is probably an important factor in the extreme delay of flowering observed in winter annuals.

Significance of FLC activity in the leaf and meristem for vernalization response

Classical experiments indicated that vernalization is perceived in the shoot apex (Curtis and Chang 1930; Melchers 1937; Lang 1965). For example, localized cooling of the apex was sufficient to induce vernalization in celery and chrysanthemum (Curtis and Chang 1930; Schwabe 1954). Although such experiments are consistent with the meristem being the site of cold perception in vernalization, localized cooling studies cannot rule out the involvement of young leaves. Indeed, in sugar beet the unvernalized meristem could be induced to flower by grafting it onto a shoot containing leaves that produce the floral stimulus (Stout 1945). Therefore, in this species, the meristem is competent to flower without vernalization. Furthermore, vernalization of sugar beet leaves that were already present prior to vernalization, and therefore not produced from a vernalized meristem, induced flowering (Crosthwaite and Jenkins 1993), demonstrating that vernalization can be detected in the leaves of this species. Perception of vernalization in the leaf was also demonstrated in experiments in which plants were regenerated from vernalized tissues. In Thlaspi arvense and Lunaria bennis, plants regenerated from leaves that were young and developing during cold exposure flowered without vernalization, whereas plants regenerated from nonvernalized leaves did not (Wellensiek 1961, 1964; Metzger 1988). These experiments indicated that cells in the leaf can respond to vernalization.

In Arabidopsis, FLC is the primary target of vernalization, and its expression is progressively repressed by longer periods of cold (Michaels and Amasino 1999; Shel- 
don et al. 1999). FLC mRNA is present in the leaf and the meristem, indicating that, in principle, FLC could repress flowering by both repressing formation of the floral stimulus and the competence of the meristem to respond to it. Our data support a dual role for FLC by showing that its expression in the meristem or the phloem delays flowering. Furthermore, vernalization of FRI FLC plants reduces FLC expression in the meristem and leaves. We propose that FLC-mediated repression is essential in the leaves to prevent formation of the transmissible signal controlled by FT. Indeed, our results indicate that repression of FLC target genes only in the meristem is probably not sufficient to repress the floral-inducing effect of the FT signal for long enough to delay flowering to an extent that would be ecologically significant (Fig. 1). Classical results from many species indicate that in inductive photoperiods, the transmissible signal is typically produced most effectively from mature source leaves, which export photosynthate through the phloem to the apex so that the floral stimulus is cotransported with photosynthate in the phloem (Zeevaart 1976, 1985). In plants in which flowering is promoted by vernalization followed by inductive photoperiods, flowering probably requires that these mature source leaves have become vernalized. The mechanism of vernalization-mediated epigenetic repression of FLC involves modifications of the histones at FLC chromatin (Gendall et al. 2001; Bastow et al. 2004; Sung and Amasino 2004). Such modifications probably require DNA replication to facilitate remodeling of the chromatin of FLC and would not be expected to occur in mature leaves that produce the floral stimulus, because their cells are no longer undergoing division. Vernalized mature source leaves could be derived from either a vernalized meristem or from young leaves that were still competent to directly become vernalized during cold exposure. Thus in certain species, the range of tissues in the apex that are competent to become vernalized will influence the length of time after vernalization required for mature floral stimulus-producing source leaves to appear. A corollary is that repression of genes such as FT and SOC1 in mature leaves would still delay flowering immediately after vernalization, until new vernalized source leaves were produced. Variation in the range of tissues that are competent to respond to vernalization and the length of time required for source leaves to be produced could contribute to differences in the vernalization response among different plant species. Furthermore, the genes that confer a vernalization response appear to differ between Arabidopsis and wheat (Yan et al. 2003, 2004), suggesting that the vernalization response evolved independently in different Angiosperm families and that the relative roles of the leaves and the meristem in the vernalization response might differ between species depending on the patterns of expression of these genes.

\section{Materials and methods}

Plant material and growth conditions

Arabidopsis plants used for transformation experiments were FRI flc-3 (Columbia, Col), in which the FRI allele from the San
Feliu-2 (SF2) accession was introgressed into Columbia (Michaels and Amasino 1999), soc1-1 (Landsberg erecta, Ler), or co-2 (Ler). Arabidopsis mutants used for flowering-time experiments $f d-1, f t-7, f d-1 f t-7$, and soc1-1 ft-7 were in the Ler background. FRI FLC and $f d$-3 (T-DNA insertion SALK_054421) were in the Col background. Plants were grown on soil in controlled environment rooms under LDs (16 h light/8 h dark) or SDs (10 h light/14 h dark) as described (Putterill et al. 1995), or on MS agar under LDs (16 h light $/ 8 \mathrm{~h}$ dark). Plants were vernalized at $4^{\circ} \mathrm{C}$ for $28 \mathrm{~d}$ under LD conditions. Flowering time was measured by scoring the number of rosette and cauline leaves on the main stem of at least 18 individuals. Data are expressed as mean \pm SE.

\section{Plasmid constructions}

GATEWAY entry clones containing the AtSUC2, AtKNAT1, AtUFO, AtML1, rolC, TobRB7, and CaMV35S promoters were described by An et al. (2004). The SOC1 and FLC cDNAs were PCR-amplified using each primer pair SOC1_F, $5^{\prime}$-CTGGTACC GATGGTGAGGGGCAAAACTCA-3' and SOC1_R, 5'-GTAC TAGTTCACTTTCTTGAAGAACAAG-3' ; and FLC_F, 5' -CCC CTCGAGCCATGGGAAGAAAAAAACTAGA-3' and FL C_R, 5'-TCCACTAGTAAGGTGGCTAATTAAGTAGTC-3', respectively. The cDNAs were fused downstream of the GATEWAY (GW) vector conversion fragment rfA in pGreen to generate binary destination vectors. Different promoter fusions were produced by LR reactions. FLC-HA fusion protein was made by amplifying the FLC cDNA with primers FLC_HA_F, 5' -CCAT GGGAAGAAAAAAACTAGAAATC-3' and FLC_HA_R, 5' -CA TTAAGTAGTGGGAGAGTCACCGG-3', flanked by GW recombination sites, and recombined into a GW entry clone. The $F L C$ cDNA was then recombined into a pJawohl binary destination vector to produce $35 \mathrm{~S}:$ :FLC-HA.

\section{Plant transformation}

All plasmids were introduced into Agrobacterium strain GV3101(pMP90) (Koncz and Schell 1986) and transformed into FRI flc-3, soc1-1, or co-2 plants by floral dip (Clough and Bent $1998)$.

\section{In situ hybridization}

Methods of digoxigenin labeling of mRNA probes, tissue preparation, and in situ hybridization were as already described (Bradley et al. 1993) with small modifications. Protease treatment was performed not with Pronase but with Proteinase K $(1 \mu \mathrm{g} / \mathrm{mL}$ in $100 \mathrm{mM}$ Tris at $\mathrm{pH} 8,50 \mathrm{mM}$ EDTA) at $37^{\circ} \mathrm{C}$ for $30 \mathrm{~min}$, and the post-hybridization washes were performed in $0.1 \times$ SSC. The probe used to detect the $F T$ transcripts was prepared from pD301 containing $450 \mathrm{bp}$ of the $5^{\prime} F T$ cDNA. Probes to detect the FD and SOC1 transcripts were PCR-amplified from cDNA using primer pairs $F D T 7-2 \mathrm{R}, 5^{\prime}$-TAATACGACTCACTATAGGG ACCAGAGCCTCGAAAGAGGT-3' and FDT3-2F, 5'-ATTAAC CCTCACTAAAGGGATTTCATCCTCATCACCATCG-3', and SOC1T3-4F, 5'-ATTAACCCTCACTAAAGGGAATCGAGGA GCTGCAACAGAT-3' and SOC1T7-4R, 5'-TAATACGACTC ACTATAGGGTTGACCAAACTTCGCTTTCA-3', respectively. The underlined nucleotide sequence indicates either the $\mathrm{T} 3$ or T7 RNA polymerase-binding sites.

\section{Analysis of FD, FLC, FT, and SOC1 mRNA abundance}

RNA was isolated from leaves using TRIZOL reagent, and DNA contamination was removed by using Ambion's DNA-free kit. RNA was analyzed by RT-PCR. For synthesis of cDNA, $2.5 \mu \mathrm{g}$ 
of total RNA was primed using dT15 primer. cDNA was diluted to $100 \mu \mathrm{L}$ with water, and $3 \mu \mathrm{L}$ of diluted cDNA was used for PCR. Amplified products were detected using SyBR Green I in a IQ5 (Bio-Rad) thermal cycler. FD was amplified using primers FD_F, 5' -CTTTTCCACCTCCTGCAACTG-3' and FD_R, 5' -CA TTTTCTGCCTGCAAGTGAG-3'. FT was amplified using primers $F T$ F, 5'-ACAACTGGAACAACCTTTGGCAATG-3' and $F T \_\mathrm{R}, 5^{\prime}$-ACTATATAGGCATCATCACCGTTCGTTACT CG-3'. Tubulin was amplified using primers described by Lee et al. (2000). SOC1 was amplified using primers SOC1_F, 5'-GAA CAAATTGAGCAGCTCAAG-3' and SOC1_R, 5'-GCAGCTT TAGAGTTTTGTTAC-3'.

\section{ChIP assays}

The 35S::HA:FLC line used flowered with $12.01( \pm 0.21)$ leaves compared with $11.08( \pm 0.18)$ for wild-type plants, and was therefore later flowering than wild-type plants $(P \leq 0.05)$. A complete description of the ChIP assays is provided in the Supplemental Material. Briefly, 10-d-old plants grown on GM agar were harvested, immersed in PBS supplemented with $1 \%$ formaldehyde, and vacuum-infiltrated for $20 \mathrm{~min}$. Glycine was added to a final concentration of $0.1 \mathrm{M}$ and incubated for $5 \mathrm{~min}$, and the seedlings were then removed from the solution and frozen in liquid nitrogen. Approximately $2.0 \mathrm{~g}$ of seedlings was ground in liquid nitrogen and resuspended in $25 \mathrm{~mL}$ of NIB. After centrifugation at $2500 \mathrm{~g}$ for $20 \mathrm{~min}$ at $4^{\circ} \mathrm{C}$, the nuclear pellet was resuspended and washed in NWB. After centrifuging, the pellet was resuspended in $1 \mathrm{~mL}$ of TE buffer supplemented with $0.5 \%$ SDS and mixed on a rotator. The chromatin was diluted with TE buffer to a final SDS concentration of $0.25 \%$. The DNA was sheared by sonication to $\sim 500-1000$-bp fragments. After centrifugation, approximately one-tenth was mixed with RIPA dilution buffer in a 2:3 ratio, and DTT, RNase A, proteinase inhibitor cocktail, and anti-HA (Sigma H6908) or anti-ratIgG (Sigma R3756) were added. After incubation overnight with rotation at $4^{\circ} \mathrm{C}$, the samples were cleared by centrifugation. A $30-\mu \mathrm{L}$ aliquot of washed ProteinA-coupled agarose beads was added to the supernatant, and the incubation continued on the rotating wheel. The agarose beads were then washed five times with $1 \mathrm{~mL}$ of RIPA buffer. Immunocomplexes were eluted from the beads two times with $200 \mu \mathrm{L}$ of glycine elution buffer, and the combined elutes were neutralized with $100 \mu \mathrm{L}$ of $1 \mathrm{M}$ Tris-HCl. Crosslinks were reversed by incubation at $37^{\circ} \mathrm{C}$ in the presence of 60 $\mu \mathrm{g} / \mathrm{mL}$ Proteinase $\mathrm{K}$ followed by at least $8 \mathrm{~h}$ of incubation at $65^{\circ} \mathrm{C}$. The DNA was purified by two successive phenol/chloroform/isoamyl alcohol extractions and ethanol precipitation. Pellets were washed with $70 \% \mathrm{EtOH}$ and resuspended in $100 \mu \mathrm{L}$ of $\mathrm{H}_{2} \mathrm{O}$, of which $4 \mu \mathrm{L}$ was used per PCR.

The primers used for the ChIP assay are described in the Supplemental Material.

\section{Acknowledgments}

The laboratory of G.C. is funded by a core grant from the Max Planck Society, and this work was partly funded by the DFG through SFB 572.

\section{References}

Abe, M., Kobayashi, Y., Yamamoto, S., Daimon, Y., Yamaguchi, A., Ikeda, Y., Ichinoki, H., Notaguchi, M., Goto, K., and Araki, T. 2005. FD, a bZIP protein mediating signals from the floral pathway integrator FT at the shoot apex. Science 309: 1052-1056.
An, H., Roussot, C., Suarez-Lopez, P., Corbesier, L., Vincent, C., Pineiro, M., Hepworth, S., Mouradov, A., Justin, S., Turnbull, C.G.N., et al. 2004. CONSTANS acts in the phloem to regulate a systemic signal that induces photoperiodic flowering of Arabidopsis. Development 131: 3615-3626.

Ayre, B. and Turgeon, R. 2004. Graft transmission of a floral stimulant derived from CONSTANS. Plant Physiol. 135: 2271-2278.

Bastow, R., Mylne, J.S., Lister, C., Lippman, Z., Martienssen, R.A., and Dean, C. 2004. Vernalization requires epigenetic silencing of FLC by histone methylation. Nature 427: 164167.

Blazquez, M.A. and Weigel, D. 2000. Integration of floral inductive signals in Arabidopsis. Nature 404: 889-892.

Borner, R., Kampmann, G., Chandler, J., Gleissner, R., Wisman, E., Apel, K., and Melzer, S. 2000. A MADS domain gene involved in the transition to flowering in Arabidopsis. Plant J. 24: 591-599.

Boss, P.K., Bastow, R.M., Mylne, J.S., and Dean, C. 2004. Multiple pathways in the decision to flower: Enabling, promoting, and resetting. Plant Cell 16: S18-S31.

Bradley, D., Carpenter, R., Sommer, H., Hartley, N., and Coen, E. 1993. Complementary floral homeotic phenotypes result from opposite orientations of a transposon at the plena locus of Antirrhinum. Cell 72: 85-95.

Clough, S.J. and Bent, A.F. 1998. Floral dip: A simplified method for Agrobacterium-mediated transformation of Arabidopsis thaliana. Plant I. 16: 735-743.

Corbesier, L. and Coupland, G. 2005. Photoperiodic flowering of Arabidopsis: Integrating genetic and physiological approaches to characterization of the floral stimulus. Plant Cell Environ. 28: 54-66.

Crosthwaite, S.K. and Jenkins, G.I. 1993. The role of leaves in the perception of vernalizing temperatures in sugar-beet. $J$. Exp. Bot. 44: 801-806.

Curtis, O.F. and Chang, H.T. 1930. The relative effectiveness of the temperature of the crown as contrasted with that of the rest of the plant upon the flowering of celery plants. Am. J. Bot. 17: 1047-1048.

Gendall, A.R., Levy, Y.Y., Wilson, A., and Dean, C. 2001. The VERNALIZATION 2 gene mediates the epigenetic regulation of vernalization in Arabidopsis. Cell 107: 525-535.

Hepworth, S.R., Valverde, F., Ravenscroft, D., Mouradov, A., and Coupland, G. 2002. Antagonistic regulation of flowering-time gene SOC1 by CONSTANS and FLC via separate promoter motifs. EMBO J. 21: 4327-4337.

Huang, T., Bohlenius, H., Eriksson, S., Parcy, F., and Nilsson, O. 2005. The mRNA of the Arabidopsis gene FT moves from leaf to shoot apex and induces flowering. Science 309: 16941696.

Imaizumi, T., Tran, H.G., Swartz, T.E., Briggs, W.R., and Kay, S.A. 2003. FKF1 is essential for photoperiodic-specific light signalling in Arabidopsis. Nature 426: 302-306.

Johanson, U., West, J., Lister, C., Michaels, S., Amasino, R., and Dean, C. 2000. Molecular analysis of FRIGIDA, a major determinant of natural variation in Arabidopsis flowering time. Science 290: 344-347.

Kardailsky, I., Shukla, V.K., Ahn, J.H., Dagenais, N., Christensen, S.K., Nguyen, J.T., Chory, J., Harrison, M.J., and Weigel, D. 1999. Activation tagging of the floral inducer FT. Science 286: 1962-1965.

Knott, J.E. 1934. Effect of a localized photoperiod on spinach. Proc. Soc. Hortic. Sci. 31: 152-154.

Kobayashi, Y., Kaya, H., Goto, K., Iwabuchi, M., and Araki, T. 1999. A pair of related genes with antagonistic roles in mediating flowering signals. Science 286: 1960-1962. 
Koncz, C. and Schell, J. 1986. The promoter of Tl-DNA gene 5 controls the tissue-specific expression of chimeric genes carried by a novel type of Agrobacterium binary vector. Mol. Gen. Genet. 204: 383-396.

Koornneef, M., Hanhart, C.J., and Van Der Veen, J.H. 1991. A genetic and physiological analysis of late flowering mutants in Arabidopsis thaliana. Mol. Gen. Genet. 229: 57-66.

Lang, A. 1965. Physiology of flower initiation. In Encyclopedia of plant physiology (ed. W. Ruhland), pp. 1380-1536. Springer, Berlin, Germany.

Lee, H., Suh, S.-S., Park, E., Cho, E., Ahn, J.H., Kim, S.-G., Lee, J.S., Kwon, Y.M., and Lee, I. 2000. The AGAMOUS-LIKE 20 MADS domain protein integrates floral inductive pathways in Arabidopsis. Genes \& Dev. 14: 2366-2376.

Levy, Y.Y., Mesnage, S., Mylne, J.S., Gendall, A.R., and Dean, C. 2002. Multiple roles of Arabidopsis VRN1 in vernalization and flowering time control. Science 297: 243-246.

McDaniel, C.N. 1985. Competence, determination, and induction in plant development. In Pattern formation (ed. G.M. Malakinski), pp. 393-411. MacMillan, New York.

Melchers, G. 1937. Die Wirkung von Genen, tiefen Temperaturenund blühenden Pfropfpartnern auf die Blühreife von Hyoscyamus niger L. Biol. Zbl. 57: 568-614.

Metzger, J.D. 1988. Localization of the site of perception of thermoinductive temperatures in Thlaspi-Arvense L. Plant Physiol. 88: 424-428.

Michaels, S.D. and Amasino, R.M. 1999. FLOWERING LOCUS $C$ encodes a novel MADS domain protein that acts as a repressor of flowering. Plant Cell 11: 949-956.

Michaels, S.D., Himelblau, E., Kim, S.Y., Schomburg, F.M., and Amasino, R.M. 2005. Integration of flowering signals in winter-annual Arabidopsis. Plant Physiol. 137: 149-156.

Moon, J., Lee, H., Kim, M., and Lee, I. 2005. Analysis of flowering pathway integrators in Arabidopsis. Plant Cell Physiol. 46: 292-299.

Mouradov, A., Cremer, F., and Coupland, G. 2002. Control of flowering time: Interacting pathways as a basis for diversity. Plant Cell 14 (Suppl): S111-S130.

Noh, Y.S. and Amasino, R.M. 2003. PIE1, an ISWI family gene, is required for FLC activation and floral repression in Arabidopsis. Plant Cell 15: 1671-1682.

Onouchi, H., Igeno, M.I., Perilleux, C., Graves, K., and Coupland, G. 2000. Mutagenesis of plants overexpressing CONSTANS demonstrates novel interactions among Arabidopsis flowering-time genes. Plant Cell 12: 885-900.

Putterill, J., Robson, F., Lee, K., Simon, R., and Coupland, G. 1995. The CONSTANS gene of Arabidopsis promotes flowering and encodes a protein showing similarities to zinc finger transcription factors. Cell 80: 847-857.

Samach, A., Onouchi, H., Gold, S.E., Ditta, G.S., SchwarzSommer, Z., Yanofsky, M.F., and Coupland, G. 2000. Distinct roles of CONSTANS target genes in reproductive development of Arabidopsis. Science 288: 1613-1616.

Schwabe, W.W. 1954. Factors affecting flowering in Chrysanthemum IV. The site of vernalisation and translocation of the stimulus. J. Exp. Bot. 5: 389-400.

Sheldon, C.C., Burn, J.E., Perez, P.P., Metzger, J., Edwards, J.A., Peacock, W.J., and Dennis, E.S. 1999. The FLF MADS box gene: A repressor of flowering in Arabidopsis regulated by vernalization and methylation. Plant Cell 11: 445-458.

Sheldon, C.C., Rouse, D.T., Finnegan, E.J., Peacock, W.J., and Dennis, E.S. 2000. The molecular basis of vernalization: The central role of FLOWERING LOCUS C (FLC). Proc. Natl. Acad. Sci. 97: 3753-3758.

Sheldon, C.C., Conn, A.B., Dennis, E.S., and Peacock, W.J. 2002. Different regulatory regions are required for the vernaliza- tion-induced repression of FLOWERING LOCUS C and for the epigenetic maintenance of repression. Plant Cell 14: 2527-2537.

Smith, H.M.S., Campbell, B.C., and Hake, S. 2004. Competence to respond to floral inductive signals requires the homeobox genes PENNYWISE and POUND-FOOLISH. Curr. Biol. 14: 812-817.

Stout, M. 1945. Translocation of the reproductive stimulus in sugar beets. Bot. Gaz. 107: 86-95.

Suarez-Lopez, P., Wheatley, K., Robson, F., Onouchi, H., Valverde, F., and Coupland, G. 2001. CONSTANS mediates between the circadian clock and the control of flowering in Arabidopsis. Nature 410: 1116-1120.

Sung, S.B. and Amasino, R.M. 2004. Vernalization in Arabidopsis thaliana is mediated by the PHD finger protein VIN3. Nature 427: 159-164.

Takada, S. and Goto, K. 2003. TERMINAL FLOWER2, an Arabidopsis homolog of HETEROCHROMATIN PROTEIN1, counteracts the activation of FLOWERING LOCUS $T$ by CONSTANS in the vascular tissues of leaves to regulate flowering time. Plant Cell 15: 2856-2865.

Teper-Bamnolker, P. and Samach, A. 2005. The Flowering Integrator FT regulates SEPALLATA3 and FRUITFULL accumulation in Arabidopsis leaves. Plant Cell 17: 2661-2675.

Valverde, F., Mouradov, A., Soppe, W., Ravenscroft, D., Samach, A., and Coupland, G. 2004. Photoreceptor regulation of CONSTANS protein and the mechanism of photoperiodic flowering. Science 303: 1003-1006.

Wellensiek, S.J. 1961. Leaf vernalization. Nature 192: 1097-1098.

- 1964. Dividing cells as prerequisite for vernalization. Plant Physiol. 39: 832-835.

Wigge, P.A., Kim, M.C., Jaeger, K.E., Busch, W., Schmid, M. Lohmann, J.U., and Weigel, D. 2005. Integration of spatial and temporal information during floral induction in Arabidopsis. Science 309: 1056-1059.

Wu, X., Dinneny, J.R., Crawford, K.M., Rhee, Y., Citovsky, V., Zambryski, P.C., and Weigel, D. 2003. Modes of intercellular transcription factor movement in the Arabidopsis apex. Development 130: 3735-3745.

Yan, L., Loukoianov, A., Tranquilli, G., Helguera, M., Fahima, T., and Dubcovsky, J. 2003. Positional cloning of wheat vernalization gene VRN1. Proc. Nat1. Acad. Sci. 100: 62636268.

Yan, L., Loukoianov, A., Blechl, A., Tranquilli, G., Ramakrishna, W., SanMiguel, P., Bennetzen, J.L., Echenique, V., and Dubcovsky, J. 2004. The wheat VRN2 gene is a flowering repressor down-regulated by vernalization. Science 303: 1640-1644.

Yoo, S.K., Chung, K.S., Kim, J., Lee, J.H., Hong, S.M., Yoo, S.J., Yoo, S.Y., Lee, J.S., and Ahn, J.H. 2005. CONSTANS activates SUPPRESSOR OF OVEREXPRESSION OF CONSTANS 1 through FLOWERING LOCUS T to promote flowering in Arabidopsis. Plant Physiol. 139: 770-778.

Zeevaart, J.A.D. 1976. Physiology of flower formation. Annu. Rev. Plant Physiol. 27: 321-348.

1985. Perilla. In CRC handbook of flowering (ed. A.H. Halevy), Vol. 5, pp. 239-252. CRC Press, Boca Raton, FL. 


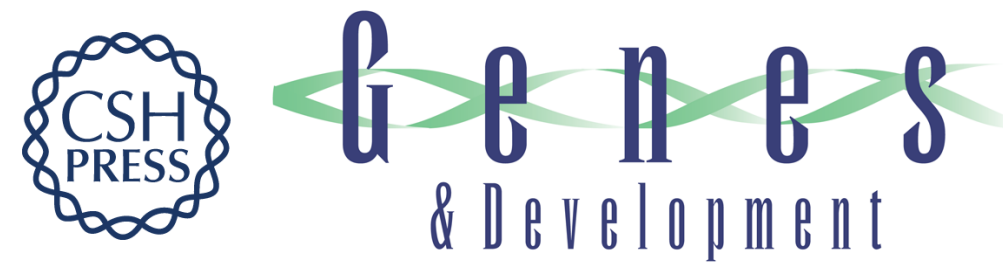

\section{The transcription factor FLC confers a flowering response to vernalization by repressing meristem competence and systemic signaling in Arabidopsis}

lain Searle, Yuehui He, Franziska Turck, et al.

Genes Dev. 2006, 20:

Access the most recent version at doi:10.1101/gad.373506

Supplemental http://genesdev.cshlp.org/content/suppl/2006/03/16/20.7.898.DC1

Material

References This article cites 53 articles, 22 of which can be accessed free at: http://genesdev.cshlp.org/content/20/7/898.full.html\#ref-list-1

License

Email Alerting Receive free email alerts when new articles cite this article - sign up in the box at the top Service right corner of the article or click here.

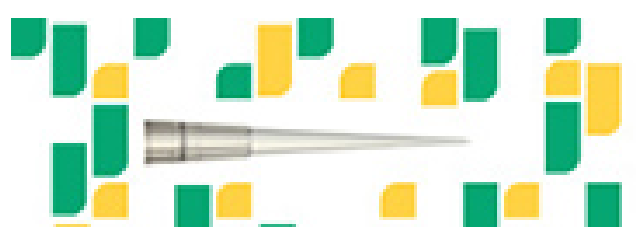

Focused on your science. 